\title{
Knowledge discovery computing for multimedia
}

Published online: 5 November 2021

๑) Springer Science+Business Media, LLC, part of Springer Nature 2021

Multimedia Tools and Applications gratefully acknowledges the editorial work of the scholars listed below on the special issue entitled "Knowledge Discovery Computing for Multimedia" (SI 1145 T).

Of 70 papers submitted, 19 were accepted for this issue after a stringent peer review process.

\section{Corresponding Guest Editor}

Roy C. Park

Sangji University, South Korea

Email: roypark@sangji.ac.kr

\section{Guest Editors}

Sunmoon Jo

Paichai University, South Korea

Email: sunmoon@pcu.ac.kr

\section{Jieun Lee}

Korea Convergence Society, South Korea

Email: jieun.lee.0072@gmail.com

\section{Supratip Ghose}

University of Information Technology \& Sciences, Dhaka, Bangladesh

Email: sgresearch@gmail.com

Publisher's note Springer Nature remains neutral with regard to jurisdictional claims in published maps and institutional affiliations. 